\title{
Transcriptomic and proteomic analyses uncover the drought adaption landscape of Phoebe zhennan
}

Na Xie ${ }^{1,2}$, Bo Li ${ }^{1,2}$, Jing Yu ${ }^{3}$, Ruxia Shi ${ }^{1,2}$, Qin Zeng ${ }^{1,2}$, Yunli Jiang ${ }^{4^{*}}$ and Dan Zhao ${ }^{1,2^{*}}$

\begin{abstract}
Background: Phoebe zhennan S.Lee (nanmu) is listed as a threatened tree species in China, whose growth and development, especially during the seedling stage, can be severely limited by drought. Previous studies on nanmu responses to drought stress involved physiological and biochemical analyses, while the molecular mechanisms remained unclear. Therefore, it is of great significance to carry out molecular biology research on the drought resistance of nanmu and reveal the genetic background and molecular regulation mechanism of nanmu drought resistance.

Results: Drought stress enhanced the soluble sugar (SS), free proline(PRO), superoxide anion (O2--), and hydrogen peroxide $\left(\mathrm{H}_{2} \mathrm{O}_{2}\right)$ contents as well as the peroxidase (POD) and monodehydroascorbate reductase (MDHAR) activities of nanmu. However, glutathione S-transferase (GST) activity was sensitive to drought stress. Further transcriptomic and proteomic analyses revealed the abundant members of the differentially expressed genes(DEGs) and differentially expressed proteins(DEPs) that were related to phenylpropanoid and flavonoid biosynthesis, hormone biosynthesis and signal transduction, chlorophyll metabolism, photosynthesis, and oxidation-reduction reaction, which suggested their involvement in the drought response of nanmu. These enhanced the osmotic regulation, detoxification, and enzyme-induced and non-enzyme-induced antioxidant ability of nanmu. Moreover, 52\% (447/867) of proteins that were up-regulated and 34\% (307/892) down-regulated ones were attributed to the increase and decrease of transcription abundance. Transcript up $\left(\mathrm{T}^{\mathrm{U}}\right)$ and protein up $\left(\mathrm{P}^{\mathrm{U}}\right)$ groups had 447 overlaps, while transcript down $\left(\mathrm{T}^{\mathrm{D}}\right)$ and protein down $\left(\mathrm{P}^{\mathrm{D}}\right)$ groups had 307 overlaps, accounting for 54\% of up and 35\% of down-regulated proteins. The lack of overlap between DEGs and DEPs also suggested that post-transcriptional regulation has a critical role in nanmu response to drought.
\end{abstract}

Conclusions: Our research results provide significant insights into the regulatory mechanisms of drought stress in nanmu.

Keywords: Phoebe zhennan, Drought, Antioxidant enzyme, Hormone, Transcriptomic, Proteomic

*Correspondence: 78185087@qq.com; danzhao_2827755@126.com

${ }^{2}$ Guizhou Academy of Agricultural Sciences, Guizhou Plant Conservation

Technology Center, Guiyang 550006, Guizhou, China

${ }^{4}$ Guizhou Academy of Forestry, Guiyang 550005, Guizhou, China

Full list of author information is available at the end of the article

\section{Background}

Drought can significantly affect the growth and development of plants throughout their whole life cycle [1]. Phoebe zhennan S. Lee (Lauraceae; Golden Phoebe) is a slow-growing forest tree whose precious timber tree species and landscaping tree species are unique to China's subtropics. Nanmu is an important timber-producing tree species that have been listed as threatened species 
by the International Union for Conservation of Nature (IUCN) and is therefore nationally protected in China [2]. In addition to human activities, plantation studies have shown that nanmu trees rarely reach their full growth potential due to abiotic stress constraints, such as drought stress. Therefore, improving the understanding of the stress tolerance mechanism of nanmu is beneficial for tree reproduction and cultivation.

Numerous studies have shown that plants develop various strategies to survive and improve their tolerance to drought under water-stressed conditions [3]. The most common strategies are morphological changes, which mainly include leaf curling. Rapid stomatal closing and increased root length are key morphological events that reduce water loss and provide greater access to water, respectively $[4,5]$. Meanwhile, a series of complex biochemical and physiological responses occur, which mainly include antioxidant enzymes, endogenous hormones, photosynthetic systems, and osmotic regulators [6]. Plants have evolved an antioxidant defense system that includes enzymatic and non-enzymatic elements present in plant cells to counteract oxidative stress $[7,8]$. These elements include accumulation of soluble proteins, SS, O2--, and $\mathrm{H}_{2} \mathrm{O}_{2}$. in cells $[9,10]$ as well as activation of reactive oxygen species (ROS), POD, catalase (CAT), and superoxide dismutase (SOD) in cells [11, 12], which are involved in the processes of water preservation and cell membrane stability. Drought has also been identified as the most severe agricultural and environmental issue globally. Plants employ efficient and sophisticated regulatory mechanisms to cope with drought stress where numerous genes and thousands of biochemical and cellular processes coordinate mediate various adaptive processes $[13,14]$. When plants are under drought stress, plants activate physiological, biochemical, metabolic, and defense systems by altering gene expression patterns $[15,16]$.

Furthermore, the hormone signaling pathways have also been identified as critical for drought stress response in plants [17]. Drought accumulates high abscisic acid (ABA) levels via up-regulation of ABA-related biosynthetic genes so as to close stomata and reduce transpiration [18]. In addition, the gene networks involved in the biosynthesis and signaling of auxin, ethylene (ET), jasmonic acid (JA), salicylic acid (SA), flavonoid, Phenylpropanoid, and brassinosteroids (BRs) are also activated under drought stress $[19,20]$. Genes related to heat shock proteins (HSPs) secondary metabolism have also been found to significantly contribute to drought stress [21].

Over recent years, the evaluation of the adaptive mechanisms in crops, model plants, and woody plants under drought stress has gained increasing attention [22, 23]. Based on the next-generation sequencing technology, several genes and pathways related to drought stress tolerance have been identified through transcriptomic and genomic techniques [24]. In addition to iTRAQ quantitative proteomic analysis, liquid chromatography-tandem mass spectrometry (LC-MS/MS) technology, and multiplex analysis of plant proteins, plant multi-omics studies under drought conditions, such as Cassava, and Agropyron mongolicum Keng have also been reported [25, 26]. These studies revealed more genes and protein pathways in response to drought stress and provided important information for systematically elucidating the mechanism network of plants in response to drought at the biological level. At present, there are few studies on the molecular mechanism of drought stress in Lauraceae plants, especially Phoebe [27, 28], whose leaves accumulated high levels of osmotic pressure, sugar, and protein, accompanied by high levels of malondialdehyde (MDA), ROS, SOD, CAT, and POD under drought stress for 30 days.

Although this study provides essential and fundamental information about nanmu response to drought, the understanding of global molecular mechanisms underlying drought tolerance is still very limited. Recently, based on the next-generation sequencing technology, the multiomics analysis has become an efficient and economical tool for the comprehensive elucidation of gene regulatory networks in numerous biological processes. Therefore, in this study, we investigated the physiological and molecular regulation mechanism of nanmu response to drought stress by analyzing the physiological and biochemical parameters. DEGs and DEPs were further identified, including the biological processes such as photosynthesis, carbohydrate metabolism, secondary metabolism, hormones biosynthesis, and signaling. The results of this study significantly further our understanding of the transcriptomic and proteomic mechanisms in drought stress response and growth regulation of nanmu seedlings.

\section{Results \\ Physiological responses of Phoebe zhennan (nanmu) under drought stress}

To estimate the dynamic of nanmu in response to drought stress, we investigated the morphological changes and physiological responses involved in water content maintenance and oxidative damage in plant cells at five timepoints $(0,4,8,12$, and $16 \mathrm{~d})$ in leaves (Fig. 1). Compared with the control 0-day drought-stressed (0d-DS), nanmu leaves were severely wilted after the 16-day droughtstress (16d-DS) (Fig. 1A). Consistently, the relative water content (RWC) rapidly decreased by $78.27 \%$ after $16 \mathrm{~d}$ of drought stress (Fig. 1B). The significant reduction of chlorophyll a (Chl a) and chlorophyll (Chlb) during stress was observed at 12 and $8 \mathrm{~d}$, respectively (Fig. 1C). In contrast, PRO and MDHAR content increased during drought 

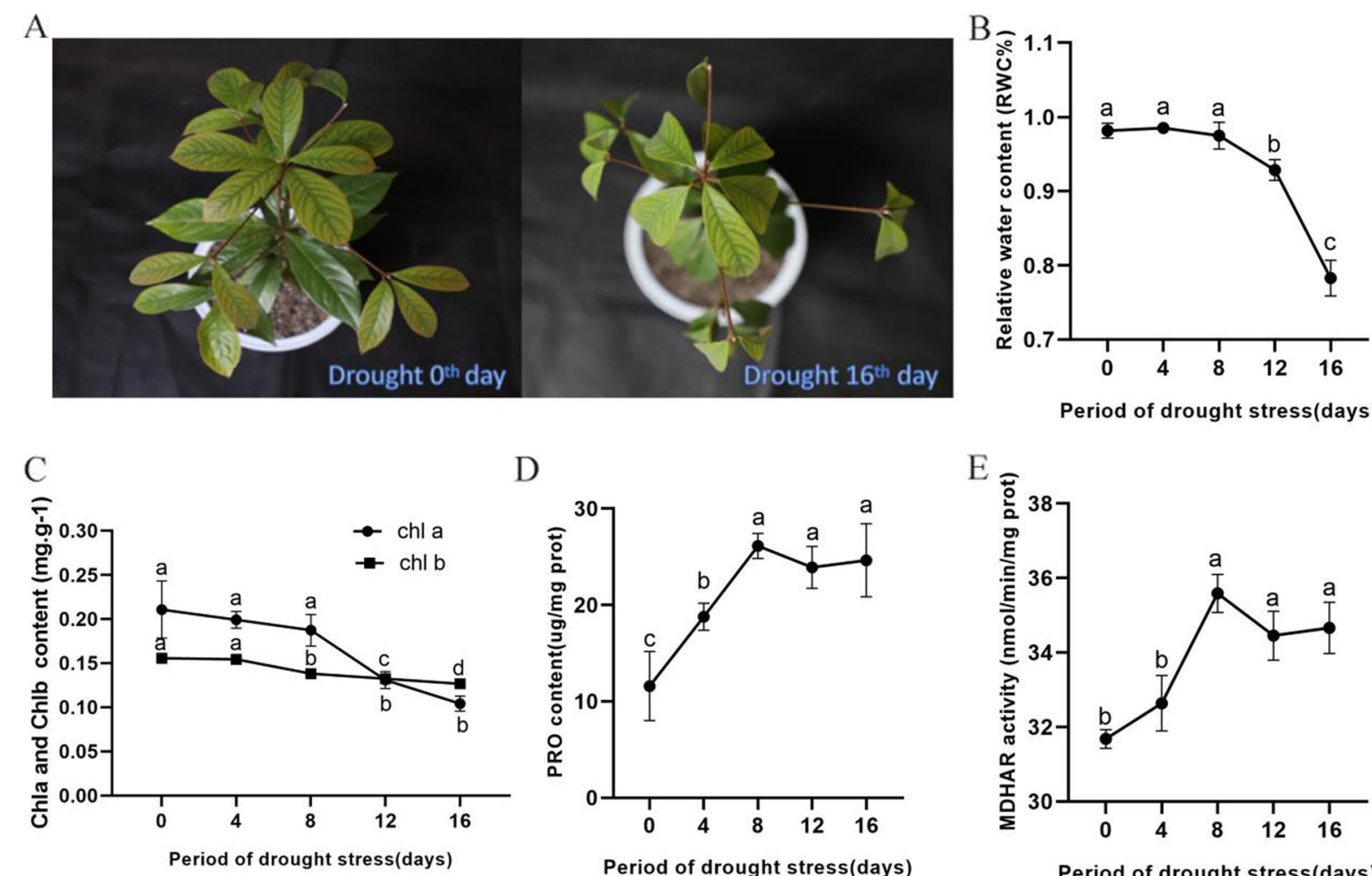

D

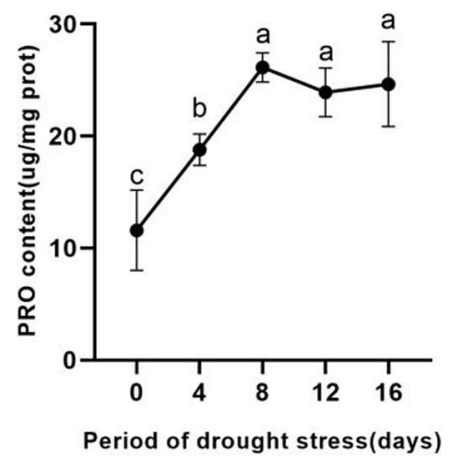

E

Period of drought stress(days)

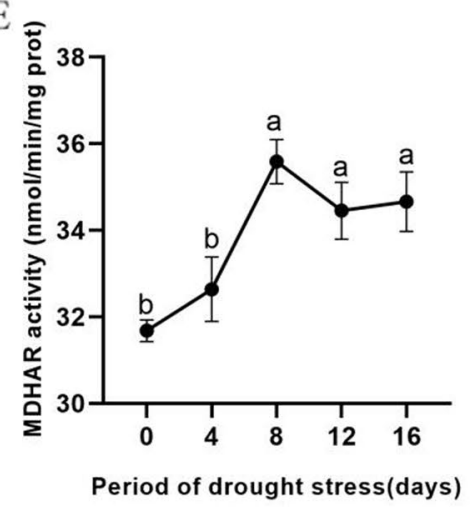

F

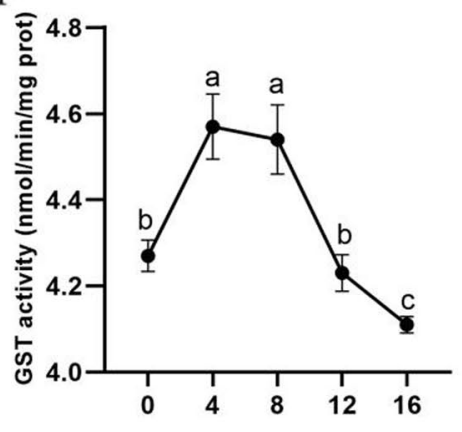

G

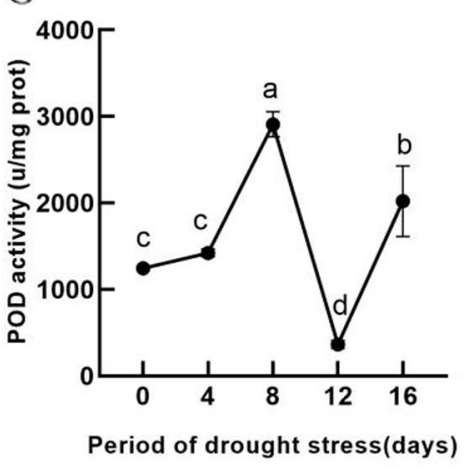

$\mathrm{H}_{\text {के }}$
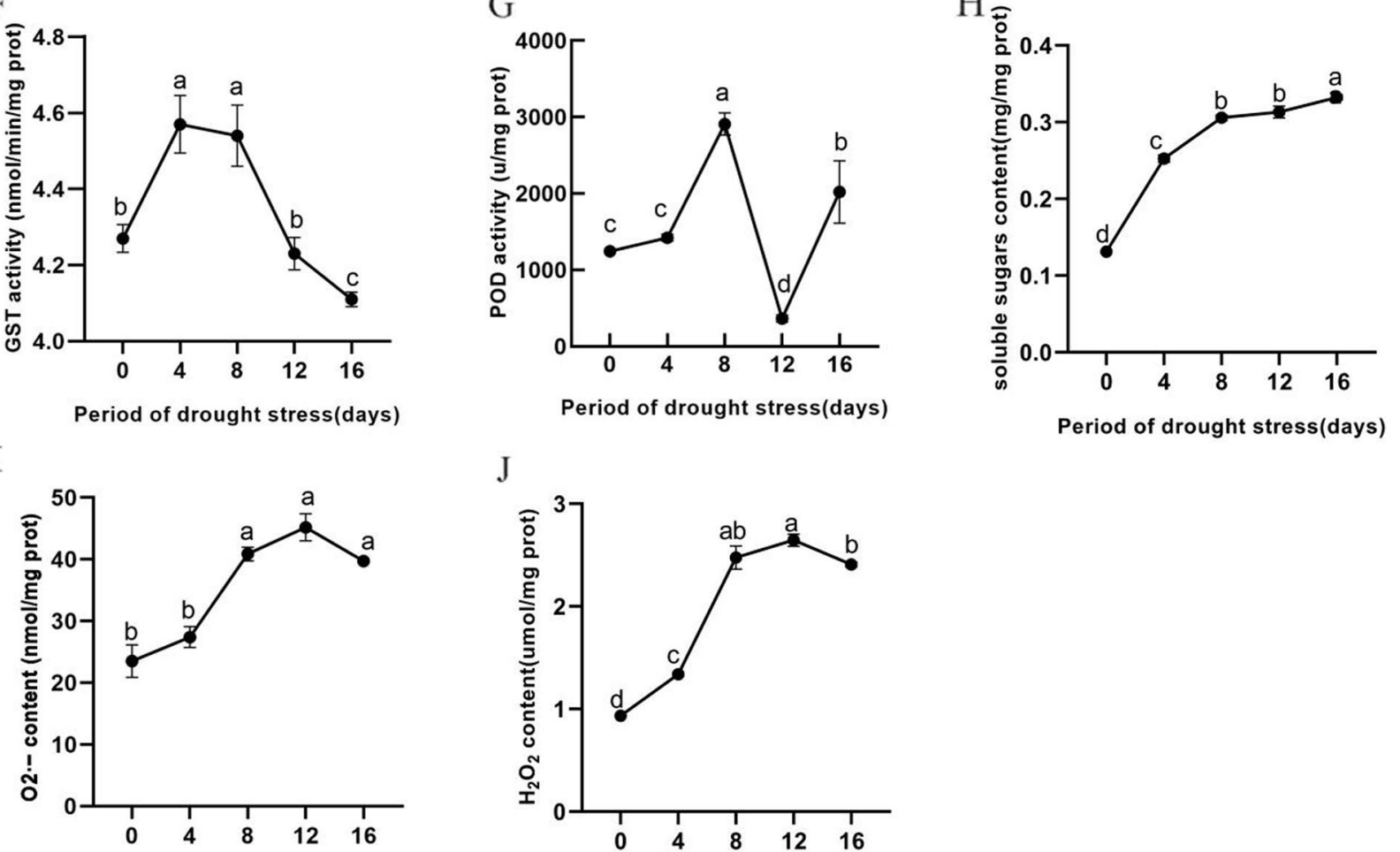

$\mathrm{J}$

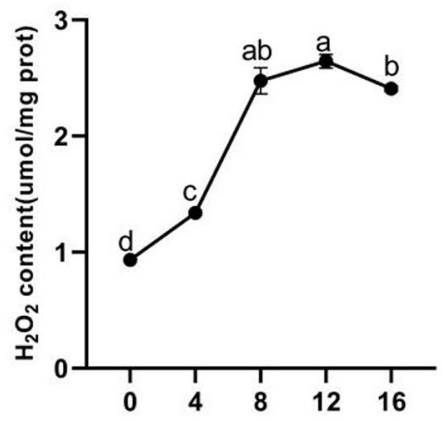

Period of drought stress(days)

Fig. 1 Physiological responses to drought stress of 0-16 days. A Nanmu plant at day 16 of water withholding (right) compared to the control plants of the same age (left). B Leaf RWC. C Chl a and b content. D PRO content. E MDHAR activity. F GST activity. G POD activity. H Soluble sugars content. I O2. - content. $\mathbf{H ~} \mathrm{H}_{2} \mathrm{O}_{2}$ content. The different letters indicate significant differences detected by the Tukey LSD test $(P<0.05)$ 


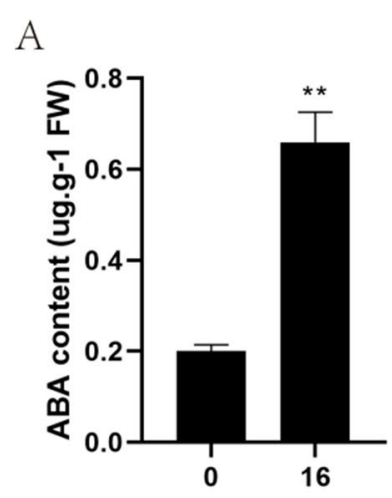

Period of drought stress(days)
$\mathrm{B}$

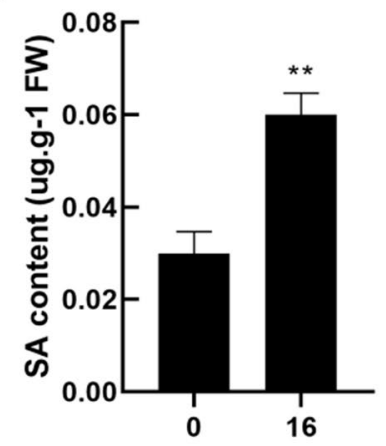

Period of drought stress(days)
C

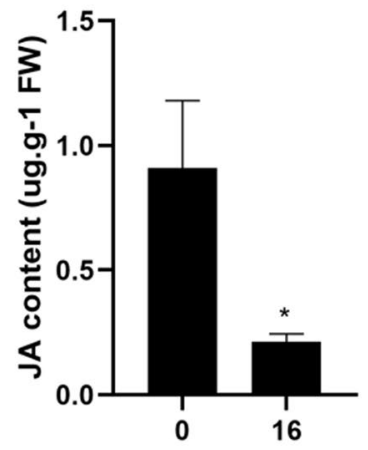

Period of drought stress(days)

Fig. 2 Hormone responses to drought stress. A ABA levels measured at 0 and $16 \mathrm{~d}$. B SA levels measured at 0 and $16 \mathrm{~d}$. C JA levels measured at 0 and 16d. Asterisks indicate significant differences detected by the Tukey LSD test $\left({ }^{*} P<0.05,{ }^{* *} P<0.01\right)$

stress (Fig. 1D, E). GST activity significantly increased from day 4 onwards and maintained this activity before the gradual decline throughout the remaining course of the experiment (Fig. 1F). The POD activity first increased and then decreased after $8 \mathrm{~d}$ of drought stress (Fig. 1G). The SS content of nanmu was found to continuously rise during drought stress (Fig. $1 \mathrm{H}$ ). The O2- - content of nanmu under drought stress did not significantly change in the early stage. It significantly increased after $8 \mathrm{~d}$, after which it finally stabilized (Fig. 1I). The content of $\mathrm{H}_{2} \mathrm{O}_{2}$ increased in the early stage and remained stable in the later stage.

We also analyzed the changes in ABA, SA, and JA in nanmu leaves under drought stress since these are commonly involved in various stresses. The ABA and SA content in nanmu leaves increased by 228.33 and $137.5 \%$ for16 d of drought stress, respectively (Fig. 2A-B). However, the JA content was reduced by $76.56 \%$ after $16 \mathrm{~d}$ of drought stress (Fig. 2C), which demonstrated that drought stress caused water loss accompanied by a series of physiological defense responses in nanmu leaves.

\section{De novo assembly and annotation of the nanmu transcriptome}

Transcriptomic analysis was performed to fully elucidate the transcriptional responses in nanmu to drought stress at 0d-DS and 16d-DS. After obtaining high-throughput reads, 63,380,859 (0d-DS) and 71,899,261 (16d-DS) clean paired-end reads were generated from RNA sequencing and used for de novo assembly (Table S2). A total of 60,250 unigenes were obtained by clustering the assembled transcripts (Table S3). More than 40,000 sequences corresponded to 200-300 bp; this number dramatically decreased when the sequence length was $>300 \mathrm{bp}$, and the total number gradually decreased as the sequence length increased (Fig. S1a). there were many sequences $>3000 \mathrm{bp}$ accounting for the high abundance (Fig. S1a). The average length of unigenes was $737 \mathrm{bp}$, and the assembled transcripts were sorted according to length (from long to short). When the length of the accumulated transcript was $50 \%$ of the total length, it corresponded to the length of the transcript (N50) of $1878 \mathrm{bp}$ (Table S3). A total of 26,541 unigenes (44.05\% of total unigenes) were matched in the five databases. Species distribution analysis showed that $71.38 \%$ of these unigenes had top matches in cinnamomum micranthum (Fig. S1b).

Subsequently, functional categorization of the unigenes with Gene Ontology (GO) was performed based on the non-redundant (NR) protein database in NCBI annotation. Unigenes were classified into 53 terms involved in biological processes, cellular components, and molecular functions (Fig. 3A). In the GO categories, "cell" or "membrane part", "catalytic activity", and "metabolic process" were dominant among all GO terms. Eukaryotic Orthologous Groups (KOG) analysis using annotated unigenes was further performed to elucidate their putative biological functions. A total of 25 functional classes were matched (Fig. S1c). The "Posttranslational modification, protein turnover, chaperones" [1527 unigenes (10.34\%)], "General function prediction only" [3039 (20.58\%)], and "Signal transduction mechanisms" [1511 (10.23\%)] represented the largest hits (Fig. S1c).

\section{DEGs enrichment and verification of transcriptome sequencing results}

We calculated the expression changes between the control and drought stress in order to identify the DEGs that responded to drought stress. The $\log 2 \mathrm{FC}<-1$ or $>1$, $(P<0.01)$ was defined as significant expression change of control versus drought stress. It was found that 4388 
and 3293 unigenes were up and down-regulated, respectively (Fig. 3B). These DEGs were significantly enriched in starch and sucrose metabolism, plant hormone signal transduction, phenylpropanoid biosynthesis, glycerolipid metabolism, flavonoid biosynthesis, alcoholism (Fig. 3C). To verify the results of the RNA-seq data, seven genes related to antioxidant enzymes, aspartate kinase, and aquaporins were randomly detected by Real-time fluorescent quantitative PCR (qRT-PCR). The obtained results were consistent with the up or down-regulation of gene expression in the transcriptomic data (Fig. S2), thus suggesting that the gene expression detected by RNA-seq is reliable.

Proteomic profiling of nanmu in response to drought stress To further understand the protein expression changes in nanmu response to drought stress, we performed a 4D label-free proteomic profiling analysis with the same conditions as the transcriptomic analysis. A total of 7051 unique proteins were identified from the 1,287,278 detected peptide spectra (Fig. S3). Expression fold (drought stress to control) was defined $>1.5$ or $<0.67$ $(p$-value $<0.05)$ as displaying a significant change. Consequently, we identified 894 up and 907 down-regulated proteins in the nanmu response to drought stress (Fig. 4A). GO, KEGG, and protein domain enrichment analysis identified 1801 DEPs. GO annotation results showed that DEPs were primarily enriched in mRNA modification and flavonol biosynthetic process in the biological processes (Fig. S4a). The DEPs were mainly concentrated in the thylakoid and chloroplast thylakoid, which is consistent with the prediction of subcellular structure and classification statistics (Fig. S4b). With reference to the molecular function, DEPs were primarily related to methyltransferase activity, hydrolase activity, acting on glycosyl bonds and structural constituent of ribosome (Fig. S4c). Kyoto Encyclopedia of Genes and Genomes (KEGG) pathway enrichment showed that many DEPs were significantly enriched in the ribosomerelated pathways, such as phenylpropanoid biosynthesis, flavonoid biosynthesis, photosynthesis, and others (Fig. 4B). In addition, protein domain enrichment analysis revealed that peroxidase, glucose-methanol-choline oxidoreductase family, and polyphenol oxidase middle domain-related proteins contained modified protein domains (Fig. S4d).

\section{Correlation of mRNA and protein profiles}

We compared the transcriptomic and proteomic data and found that 6715 proteins were quantitatively detected (Fig. 5A). Correlation analysis revealed low correlation coefficients in leaves $(R=0.54)$ (Fig. S5) between the expression levels of all quantified proteins and their corresponding mRNAs. The DEGs and the DEPs with significant changes in expression were further compared. As shown in the Venn diagram (Fig. 5B), transcript up $\left(\mathrm{T}^{\mathrm{U}}\right)$ and protein up $\left(\mathrm{P}^{\mathrm{U}}\right)$ group had 447 overlaps and transcript down $\left(\mathrm{T}^{\mathrm{D}}\right)$ and protein down $\left(\mathrm{P}^{\mathrm{D}}\right)$ group had 307 overlaps, which accounted for 54\% up and 35\% down-regulated proteins, respectively. These results suggested that the transcriptional activity of these genes may modulate their protein levels. In contrast, the $46 \%$ up and the $65 \%$ down proteins, including the $38 \mathrm{~T}^{\mathrm{D}} \mathrm{P}^{\mathrm{U}}$ overlaps and $24 \mathrm{~T}^{\mathrm{U}} \mathrm{P}^{\mathrm{D}}$ overlaps, highlighted a critical role of post-transcription modification in regulating protein expression. It is noteworthy that one gene (TRINITY DN5471_c0_g1) that was up-regulated corresponded to two proteins (TRINITY_DN5471_c0_g1_m.20867, TRINITY_DN5471_c0_g1_m.20868) that were up and down-regulated, respectively, which may be caused by alternative splicing. Meanwhile, in order to study the effect of post-transcriptional regulation, the GO functional enrichment and KEGG pathway analyses were performed (Fig. S6). The pathways that were primarily enriched included oxidoreductase activity, metabolic pathways metabolism, phenylpropanoid biosynthesis, hormone biosynthesis, and photosynthesis.

\section{The critical DEGs and DEPs related to nanmu response to drought stress}

Based on the integrative proteomic and transcriptomic analyses, we focused on critical proteins and genes implicated in several aspects of physiological and biochemical responses to drought stress. These up-regulated genes/ proteins were enriched in phenylpropanoid biosynthesis, metabolic pathways, and photosynthesis (Fig. S6). Consistently, cinnamoyl-CoA reductase 1(CCR1), phenylalanine ammonia-lyase 3 (PAL3), and cinnamyl

\footnotetext{
(See figure on next page.)

Fig. 3 GO classification, Volcano map and. KEGG pathway (A) GO secondary classification statistical distribution diagram. Note: ordinate (left), the percentage of the number of genes; ordinate (right), the number of genes aligned. B Differentially expressed volcano map. Note: Each point in the differential expression volcano plot represents a gene; log2FC: the logarithm of the fold difference in expression of a gene in two samples; $-\log 10 F C(F D R)$ : the negative logarithm of the statistical significance of the change in gene expression. The green dots in the figure represent down-regulated DEGs, the red dots represent up-regulated DEGs, and the blue dots represent non-DEGs. C DEGs KEGG pathway enrichment scatterplot. Note: vertical axis: pathway name; horizontal axis: Rich factor (the ratio of the Sample number of DEGs to the background number of DEGs). The size of the dot indicates the number of DEGs in this pathway, and the color of the dot corresponds to different Qvalue ranges. Qvalue is the $P$ value after correction for multiple hypothesis testing
} 


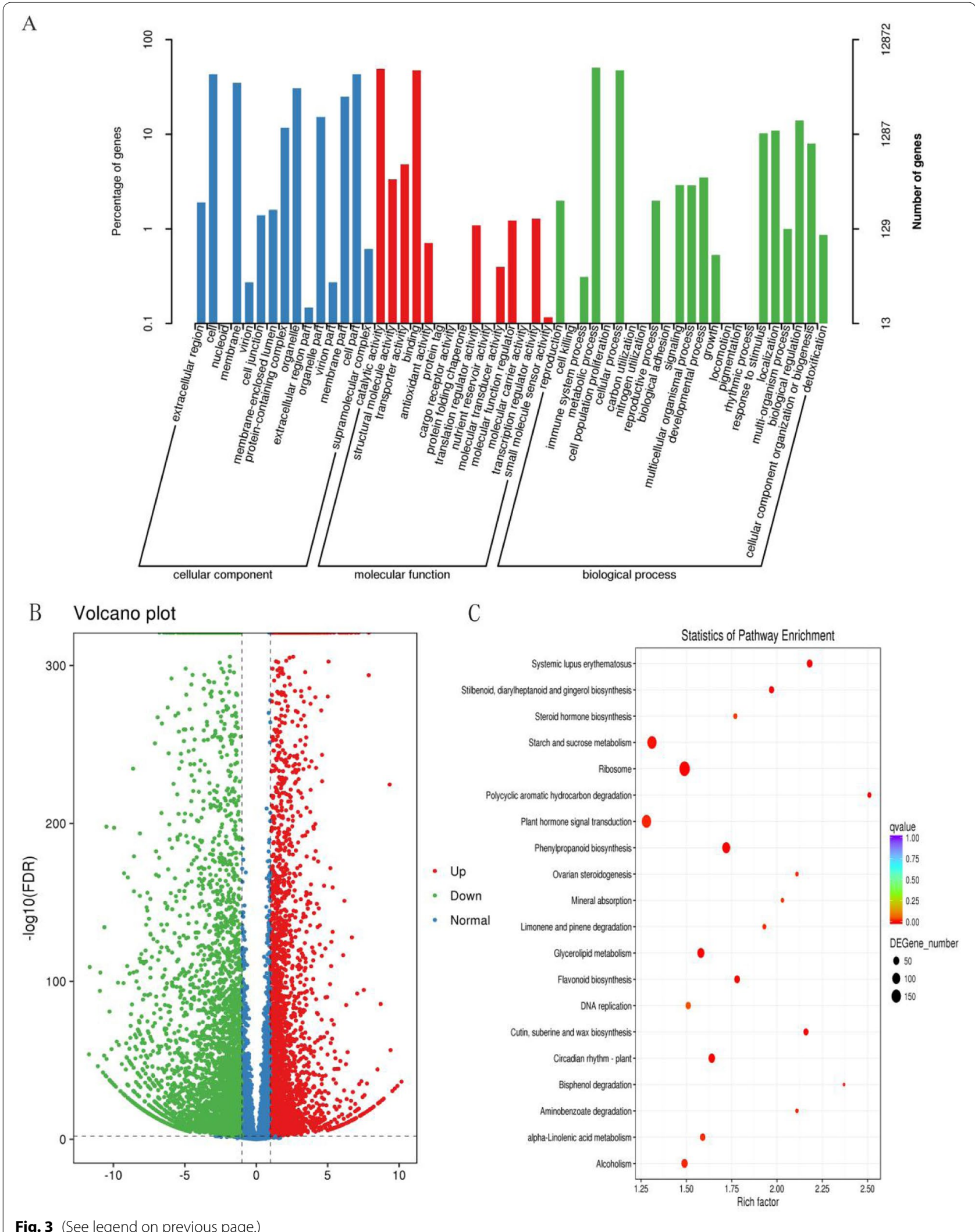

Fig. 3 (See legend on previous page.) 
alcohol dehydrogenase 1(CAD1) were involved in the phenylpropanoid biosynthesis (Table S4 and Fig. 6). Catalase isozyme 1 (CAT1) and glutamate decarboxylase 1 (GAD1) were involved in the metabolic pathways (Table S4). Drought significantly induced proteins involved in photosynthesis and photosynthesis-antenna proteins, including Photosystem II (PSBA, PSBQ), Photosystem I (PSAE, PSAL). Light-harvesting chlorophyll protein (LHCA1, LHCA3, LHCB3) was significantly up-regulated at the transcript level and protein level. Besides three genes, peroxidase (POD4, POD5, POD64, and $P O D 72$ ) that were involved in ROS scavenging were also significantly up-regulated (Table S4). Drought also greatly induced the expression of genes associated with ABA biosynthesis and Signal transduction, including protein phosphatase $2 \mathrm{C}$ (PP2C66), Serine/threonine-protein kinase (Snrk3 and Snrk2), and zeaxanthin epoxidase $(Z E P)$. These down-regulated genes were enriched in flavonoid biosynthesis (Fig. 6). chalcone--flavonone isomerase 3 (CHI3), flavanone 3-dioxygenase $(F 3 H)$, flavonoid 3 'monooxygenase $\left(F 3^{\prime} H\right)$, and leucoanthocyanidin dioxygenase $(A N S)$ were found to be involved in flavonoid biosynthesis (Table S4).

Next, we focused on the genes/proteins that only changed at the transcriptomic or proteomic level in specific pathways. There were 2841 up-regulated and 4043 down-regulated DEGs whose corresponding proteins were not differentially expressed (Fig. 5). Drought also significantly induced the expression of abiotic stress genes. Phosphatase $2 \mathrm{C}$ (PP2C 10,PP2C70, and PP2C 16), Indole-3-acetaldehyde oxidase ( $A A O 3)$, Secoisolariciresinol dehydrogenase $(A B A 2)$, carotenoid cleavage dioxygenase $(N C E D)$ were involved in the ABA biosynthesis and Signal transduction (Fig. 6). Furthermore, most of these genes were associated with HSPs (HSP17.3-B, HSC-2, HSP70, and HSP90-5) (Table S4 and Fig. 6).

There were 381 up-regulated and 560 down-regulated DEPs whose corresponding genes were not differentially expressed (Fig. 5). As expected, drought significantly induced proteins involved in photosynthesis, including Photosystem I reaction center subunit II (PSAD). Late embryogenesis abundant protein Lea14-A (LEA) protein was significantly up-regulated at the protein level (Table S4). Drought also significantly and greatly induced the proteins involved in redox-related Superoxide dismutase
(SODCC, SODCP) response to oxidative stress. On the contrary, it dramatically suppressed the expression of Glutathione S-transferase (GSTF9, GSTF17) (Table S4).

\section{Discussion \\ Physiological responses to drought stress in nanmu}

Drought is considered to be the main abiotic environmental stress that is not conducive to plant survival and growth [29]. Plant adaptation to drought stress involves a series of physiological changes [30,31]. RWC is generally considered as an indicator of plant cells' water status under drought stress, whose variation reflects the drought resistance of the plant [32,33]. Under abiotic stress conditions, excessive free radicals are formed in plants, adversely affecting plant growth and development. ROS, such as $\mathrm{H}_{2} \mathrm{O}_{2}$ and $\mathrm{O} 2 \cdot-$, are some of the most damaging molecules in plants [34]. In this work, the significant increase observed in $\mathrm{H}_{2} \mathrm{O}_{2}$ and $\mathrm{O} 2$. - levels under drought stress was consistent with the results of previous studies, thus indicating that ROS scavenging may be related to the drought response of nanmu. At the same time, under adverse conditions, antioxidant enzymes (CAT, SOD, and POD) could scavenge excess free radicals, which prevents free radicals from damaging the plant cell membrane system and other macromolecules and increases the plant's resistance to stress via antioxidant enzyme system activation [35]. Under drought conditions, plants accumulate osmotic adjustment substances such as PRO and soluble sugars to maintain cell turgor, cell membrane stability, and protein function and promote photosynthesis [36]. In the present work, the SS and Pro contents followed similar trends during the drought period, revealing a significant increase. These results were consistent with previous research [37], indicating that SS and Pro have a key role in drought tolerance.

In this study, physiological data were investigated in nanmu leaves at five-time points (within 16 days) under drought stress. This study is similar to previous studies on the physiological, osmotic regulation, and antioxidant defense systems of nanmu [28]. The physiological and biochemical indicators of these studies (including PRO content and MDHAR, GST, and POD activity) revealed the induction of response to drought stress in nanmu. During drought stress, $P$. communis and $P$. scoparia

\section{(See figure on next page.)}

Fig. 4 DEPs and KEGG pathway enrichment. A Histogram of DEPs. Note:the red represent up-regulated proteins, The green in the figure represent down-regulated proteins. Vertical axis: number of proteins. Horizontal axis: Compared sample name (16-DS/0-DS) when the $P$-value $<0.05$, the change of differential expression was more than 1.5 as the threshold of significant up-regulation, and less than 1/1.5 as the threshold of significant down-regulation. B DEPS KEGG pathway enrichment. Note: On the vertical axis of the bubble plot is a functional classification or pathway, and on the horizontal axis is a log2 converted value of the ratio of the differential protein in that functional type to the ratio of the identified protein. The color of the circle indicates the enrichment significance $p$-value, and the size of the circle indicates the number of different proteins in functional classes or pathways 


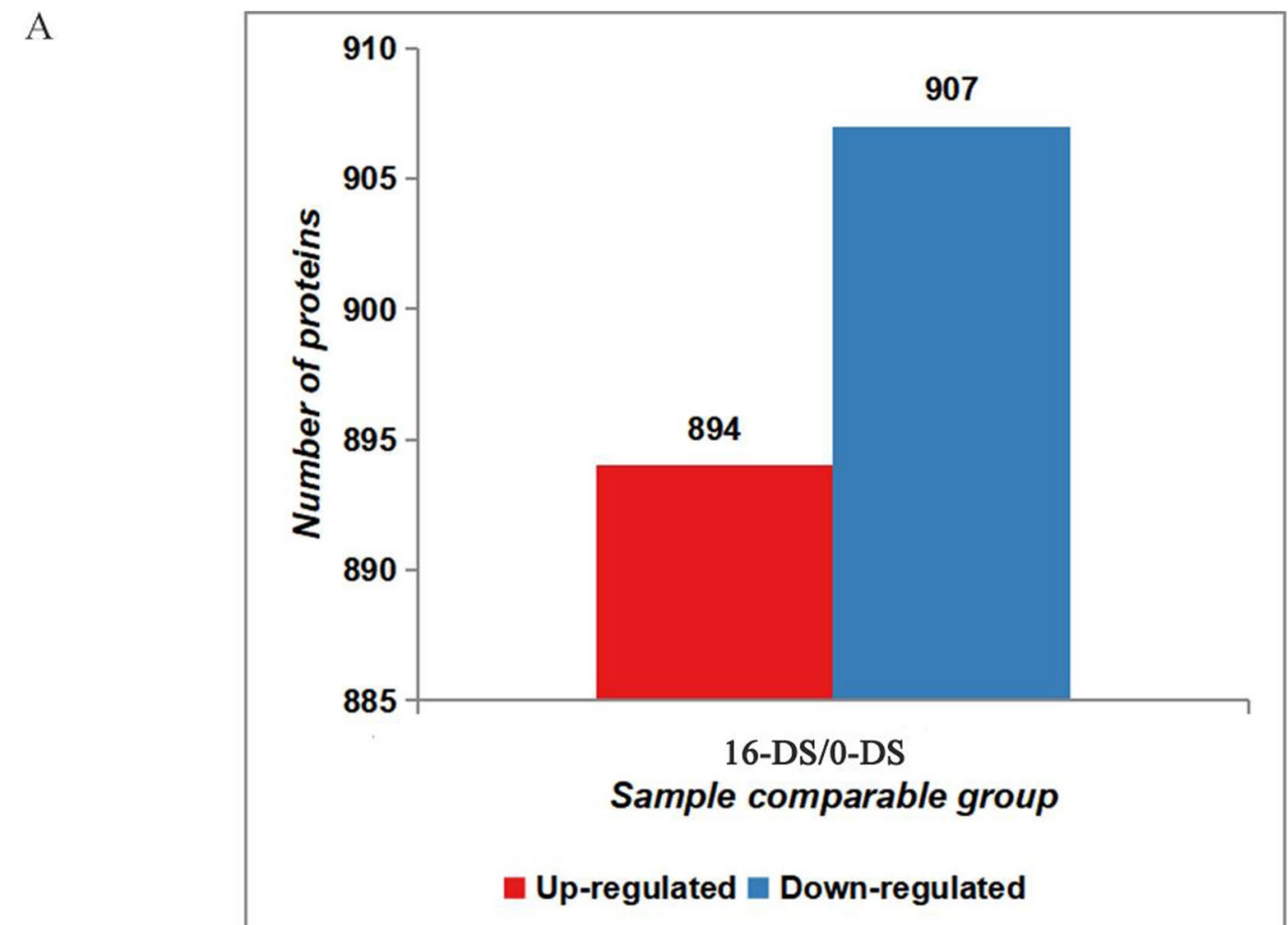

B

map00196 Photosynthesis - antenna proteins
map00902 Monoterpenoid biosynthesis map00902 Monoterpenoid biosynthesis p00603 Glycosphingolipid biosynthesis - globo and isoglobo series map00300 Lysine biosynthesis map00941 Flavonoid biosynthesis map00195 Photosynthesis map00565 Ether lipid metabolism map00940 Phenylpropanoid biosynthesis map00945 Stilbenoid, diarylheptanoid and gingerol biosynthesis map03430 Mismatch repair map00670 One carbon pool by folate map03020 RNA polymerase map00650 Butanoate metabolism map03010 Ribosome map00260 Glycine, serine and threonine metabolism map03030 DNA replication
Protein number

66

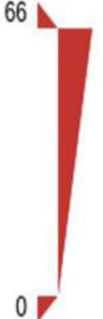

0

Pvalue 0.0419 0.0000

Fig. 4 (See legend on previous page.) 
A

Transcript

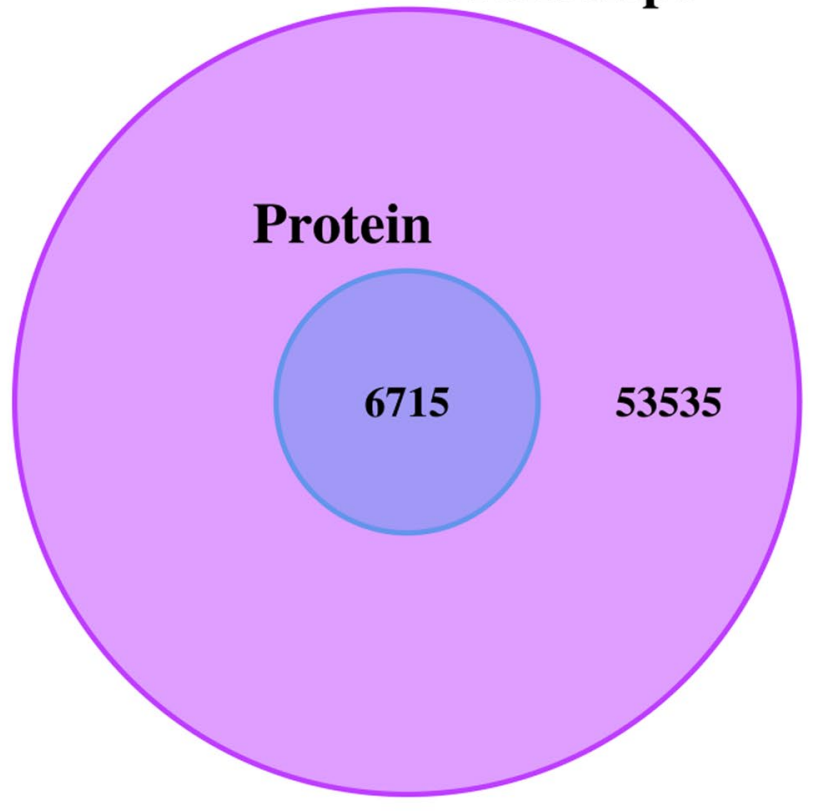

$\mathrm{B}$

\section{Transcript Transcript}

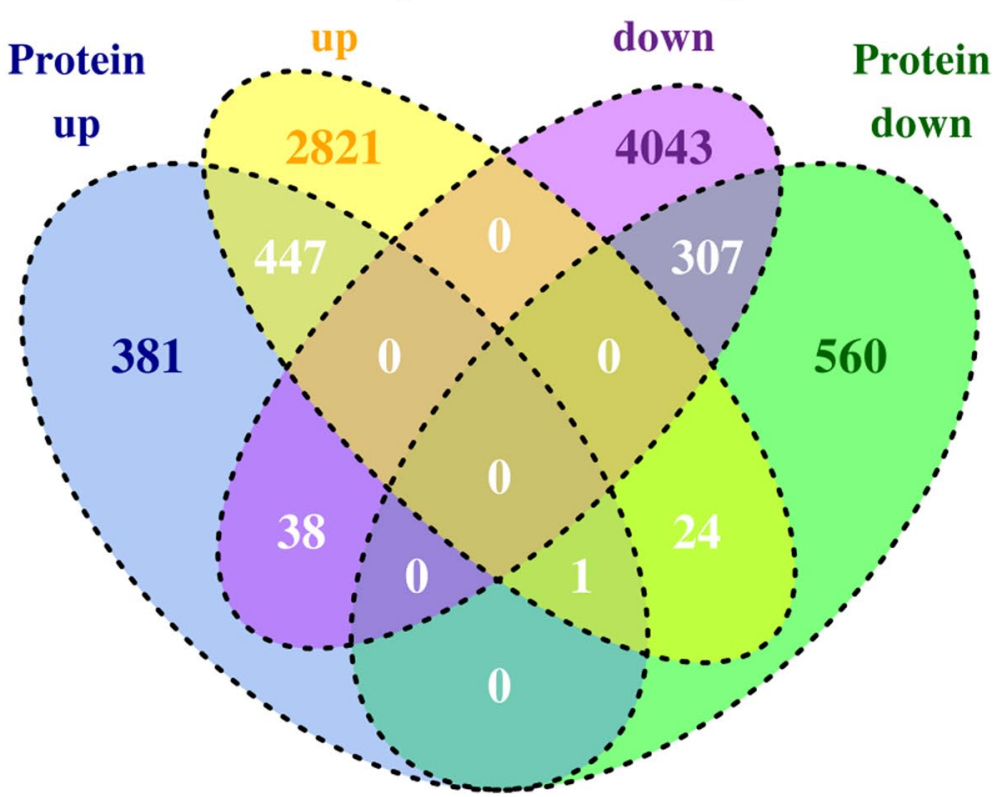

Fig. 5 Conjoint analysis Venn diagram. A Venn diagram for the quantitative comparison of the transcriptome and proteome. B Venn diagram compared between the DEPs and DEGs in the comparison group

showed both high APX and MDHAR activities [38]. In this work, the MDHAR activity value significantly increased in the middle of the drought and remained stable in the later period (Fig. 1E), which indicated that MDHAR is likely involved in the drought response in nanmu. GSTs are multifunctional enzymes that have a 


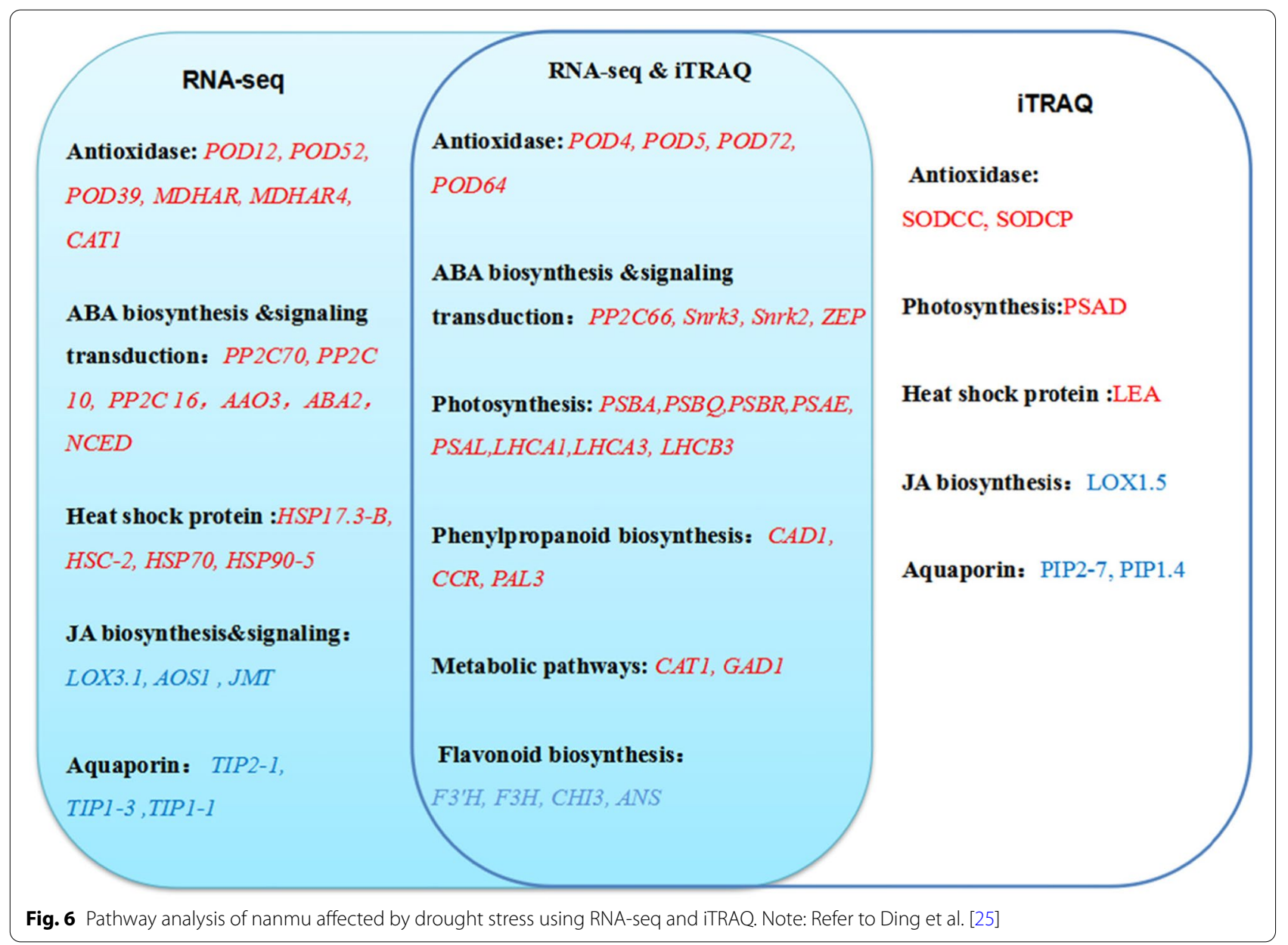

crucial role in cellular detoxification and oxidative stress tolerance [39]. In the present study, GST activity first increased and then decreased, which indicated that GST participated in the response under drought conditions. POD is one of the most important enzymes that further convert $\mathrm{H}_{2} \mathrm{O}_{2}$ into $\mathrm{H}_{2} \mathrm{O}$ and $\mathrm{O} 2$. Also, the damage caused by ROS is eliminated from plants [40]. In this study, POD activity increased in the pre-drought period, which might be a form of adaptation for alleviating oxidative stress. Subsequently, POD activity sharply decreased. These results indicated that POD is sensitive to abiotic stress and may be important for the drought tolerance mechanism of nanmu seedlings.

\section{Chlorophyll metabolism and photosynthesis}

Drought stress can lead to plant dehydration and inhibit plant growth by hindering the physiological and metabolic processes of nanmu leaves. Magnesium-chelatase subunit (ChlH, ChlD) protein was found to be downregulated under drought stress in the present study (Table S4). It is one subunit of the magnesium-chelatase complex, which has an essential role in chlorophyll biosynthesis. One of the inhibitory effects of drought on plant photosynthesis is decreased chlorophyll content in leaves [41]. In this study, Chl a and b of nanmu were significantly reduced after the $16 \mathrm{~d}-\mathrm{DS}$ treatment, which may be attributed to the down-regulation of $\mathrm{ChlH}$ and ChlD (Fig. 1C). The activity of Pheophorbide a oxygenase (PAO), a key chlorophyll catabolic enzyme, was enhanced by drought stress (Table S4).

In addition, photosynthesis and photosynthesisantenna proteins were both up-regulated (Table S4), whereas one gene involved in the photosynthetic electron transport, Ferredoxin-3(PETF), was down-regulated (Table S4). The results showed that drought stress induced the expression of genes and proteins, including PSII and PSI, which was conducive to stabilize the structure of PS II and PSI from being damaged by drought stress, repair the damaged PSI and PSII, and stabilize the redox state of photosynthetic electron transport chain. However, the significant down-regulation of PETF gene inhibited the activity of photosynthetic electron 


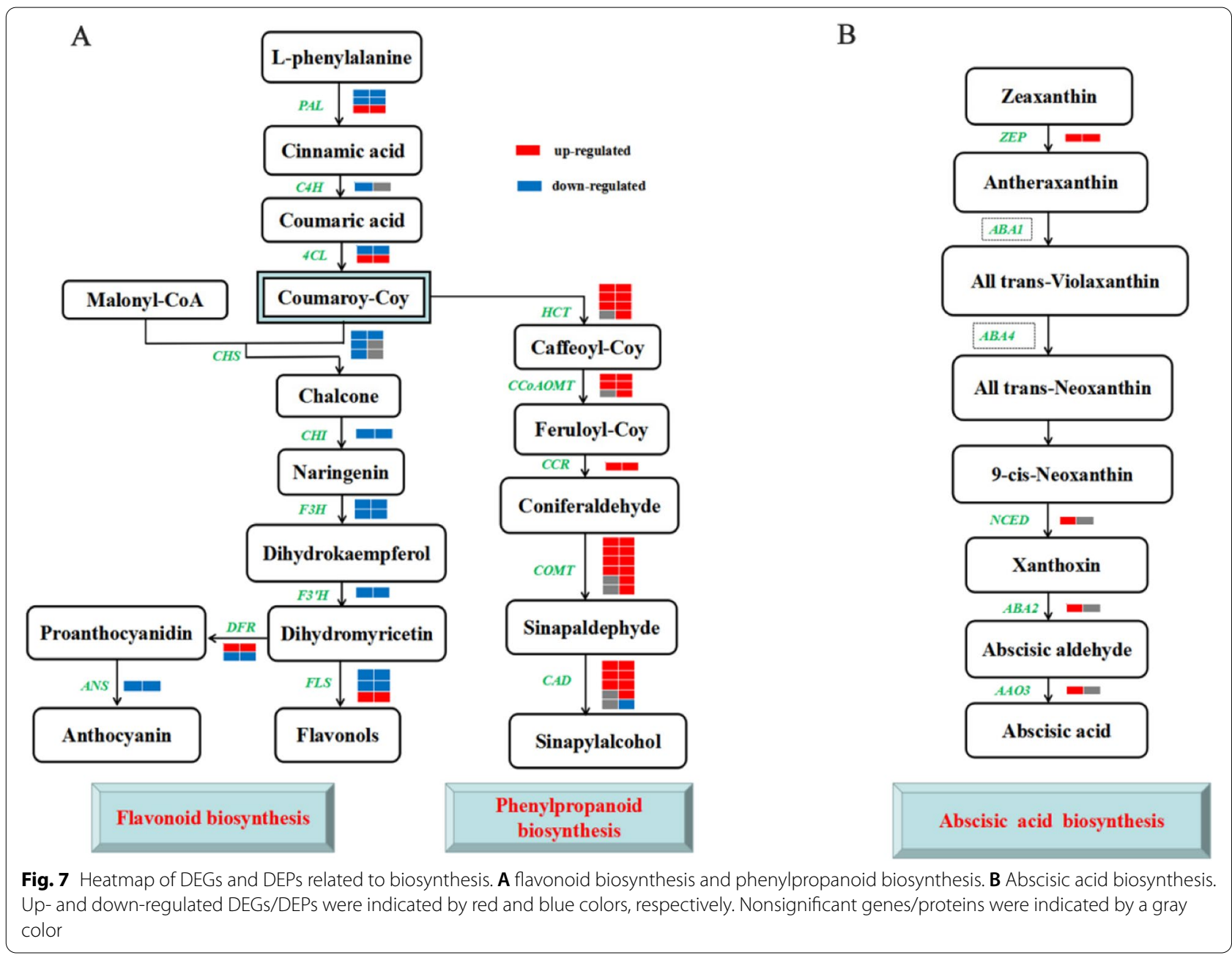

transport chain. Drought stress not only affected the light reaction process, but also affected the dark reaction process. This study found that the down-regulation of glyceraldehyde 3-phosphate dehydrogenase (GAPDH) in Kelvin pathway may lead to the decrease of photosynthetic carbon assimilation efficiency (Table S4), and then affect the photosynthetic activity of nanmu. Drought stress induced up-regulated expression of light response related proteins, which may be a mechanism of light protection and light adaptation of Phoebe to drought adverse environment.

\section{Roles of flavonoid and phenylpropanoid biosynthesis genes in drought}

Secondary metabolites such as phenylpropanoids, anthocyanins, and flavonoids are important compounds essential for plant acclimation [42]. Water deficit can significantly affect the expression of genes related to phenylpropanoid and flavonoid pathways [17]. The phenylpropanoid pathway serves as a source of metabolites in plants, as well as a starting point for the lignans and flavonoids [42]. In the present study, 13 and 25 genes were identified for flavonoid and phenylpropanoid biosynthesis pathways, respectively (Fig. 7A). Most genes in the flavonoid pathway was down-regulated and phenylpropanoid pathway was significantly up-regulated at the mRNA and protein levels, respectively. thus indicating that these genes were mainly affected by transcriptional regulation (Table S4). These results also suggested that integrative transcriptomic and proteomic analysis might be important for the investigation of the expression of flavonoid and phenylpropanoid biosynthesis genes, at least under the condition of water deficit in nanmu.

\section{The role of hormone biosynthesis in drought}

ABA, JA, and SA are considered important signals in abiotic stress response and drought stress memory [43, 44]. As far as an abiotic stress response to plant hormones, high ABA content is an important indicator of drought, high temperature, high salt, and cold $[45,46]$. 
In this study, the ABA content in nanmu leaves showed a sharp upward trend with the continuous drought stress (Fig. 2A). The increased ABA content in nanmu leaves facilities stomatal movement to reduce transpiration water loss and maintain cell viability [47], which is consistent with the previously published results [48]. Plants synthesize abscisic acid biosynthesis (ABA) using the carotenoid pathway. Zeaxanthin epoxidase $(Z E P)$ catalyzes the conversion of zeaxanthin to alltrans-violaxanthin via antheraxanthin [49], after which 9 -cis-neoxanthin can be oxidatively cleaved by the 9-cisepoxycarotenoid dioxygenase (NCED) [50]. Genetic and biochemical studies revealed that the cleavage of 9-cis-xanthophylls produces a $\mathrm{C}_{15}$-apocarotenoid and xanthoxin [51]. A short-chain alcohol dehydrogenase encoded by AtABA2/AtGIN1 then converts xanthoxin into abscisic aldehyde, which is eventually oxidized to ABA by AtABA3, an abscisic aldehyde oxidase (AAO3) [52] (Fig. 7B). These data revealed an intact biosynthesis pathway of $A B A$. In the present study, the identified synthesis-related genes, including ZEP, NCED, ABA2, and $A A O 3$ in ABA synthesis, the gene expression levels of $Z E P, N C E D, A B A 2$, and $A A O 3$ were significantly upregulated in nanmu (Table $S 4$ ). The up-regulated genes in this study were consistent with the results of $\mathrm{X} \mathrm{U}$ et al. [53], which suggested they acted as important factors in core ABA biosynthesis (Fig. 7B) Normally, ABA is involved in drought signal transduction in plants, and the expression of many genes is altered (repressed or induced) under drought conditions. In this work, the gene expression levels of Serine/threonine-protein kinase (Snrk2, Snrk3), abscisic acid receptor PYL6 (PYL), and protein phosphatase $2 \mathrm{C}$ (PP2C) were significantly upregulated in nanmu (Table S4). PP2Cs have an important role in core ABA signaling pathways [54]. PYLs have differing binding properties with $\mathrm{ABA}$, and selectively interact with PP2Cs [55]. In the absence of ABA, PP2Cs interact with and repress SnRK2s to block ABA signaling [56]. Interestingly, the expression changes of these ABA signaling genes were consistent with the increase in ABA levels, strongly supporting their involvement in ABA-dependent drought response in nanmu. Moreover, aquaporins contribute to ABA-triggered stomatal closure through OST1-mediated phosphorylation [57]. Arabidopsis AtPIP2;1 and AtPIP2;2 are down-regulated under drought stress [58]; however, AtPIP1;3, AtPIP1;4, and few other AtPIPs are up-regulated [59]. Grondin et al. found that the expression of PIP2;1, PIP1;3, PIP2;2, PIP1;1, PIP1;2, and PIP2;8 were significantly reduced in the roots of six rice varieties under drought stress. In this study, the majority of the PIP genes were down-regulated under drought stress (Fig. 6 and Table S4), which is consistent with the results reported by Grondin et al. in their study [57].

JA is essential to signal transducers that drive the expression of plant defense genes in response to environmental stress [60]. JA is extensively found in higher plants and can significantly enhance plant resistance to insects, diseases, and drought. Dong et al. [61] found that external application of methyl jasmonate (MeJA) moderately reduced the damage to rice seedlings caused by drought stress. $\mathrm{Xu}$ and colleagues [53] have pointed out that JA immediately accumulates when millet is under drought stress, and the cell turgor pressure decreases; however, JA cannot regulate water stress for an extended period of time [53], which is different from the adversity response hormone ABA. Dhakarey et al. [62] used JA to synthesize the CPM2-deficient mutant in rice and found that under drought stress, the mutant had higher efficiency of water use, stronger metabolism, and significantly increased secondary metabolites, thus suggesting that endogenous JA was a negative regulator under drought stress. In the present study, the JA content in nanmu leaves decreased after the 16-DS treatment. Therefore, based on the current experiments, it was not possible to determine whether there is a transient accumulation effect of JA, so further continuous measurement experiments are required. Yet, the identified synthesis-related genes, including Allene oxide synthase 1(AOS1) and linoleate 13S-lipoxygenase 3-1 (LOX3.1) in JA synthesis, were upregulated in transgenic levels (Fig. 6 and Table S4).

Compared with these two hormones, the SA effect under drought stress has been rarely reported. Our results revealed that SA content in nanmu leaves increased after the 16-day drought treatment. This was related to the up-regulation of the key enzymes responsible for the SA biosynthesis, including mitogen-activated protein kinase 4 (MPK4), caffeic acid 3-O-methyltransferase 1 (OMT1), caffeic acid 3-O-methyltransferase (COMT1), transcription factor TGA4 (TGA4), and caffeic acid 3-O-methyltransferase 1 (HOMT1) (Table S4). Further investigation is needed to determine whether the SA response is related to drought resistance.

\section{Roles of HSPs in drought stress}

HSPs have an important role not only in heat stress but also under other stressful conditions, including drought, salt, oxidative stress, and cold [63]. These proteins have essentially been implicated in the innate immunity and abiotic stress tolerance in higher plants. HSPs usually act as molecular chaperones to protect cells against multiple stresses through signaling. At the same time, HSPs can also function via protein folding [63]. In the present study, it was clearly observed that the majority of HSPs, such as HSP17.3-B, HSC-2, HSP70, and HSP90-5, whose 
corresponding proteins were not differentially expressed in nanmu, were dramatically up-regulated (Table S4).

It has been suggested that HSPs are involved in drought stress through transcriptional regulation. Hsp70 is involved in stomatal closure and regulation, transcriptional and physiological regulation in response to $A B A$ signaling [64]. HSPs can also participate in ROS scavenging genes to protect plants from drought stress. Furthermore, late embryogenesis abundant protein Lea14-A (LEA) protein was significantly up regulated at the protein level (Table S4). Some scholars have shown that the expression of LEA is related to the enhanced tolerance of plants to abiotic stress [65]. Xiao et al. [66] found that overexpression of LEA-related genes in rice could significantly improve plant drought resistance, suggesting that these proteins may protect biological macromolecules and prevent water loss in plant cells.

\section{Conclusion}

In this study, physiological and biochemical indices were analyzed, and transcriptomic and proteomic analyses were performed on nanmu seedlings treated with natural drought stress. We found that nanmu seedlings could regulate the antioxidant system, osmotic regulation, hormone levels, chlorophyll metabolism, photosynthesis pathway, and photosynthesis-antenna proteins, thus alleviating the damage caused by drought stress. Furthermore, we screened and identified the key metabolic pathways, genes, and proteins of nanmu that alleviate drought stress. Our results revealed that drought stress could induce nanmu oxidoreductase activity, metabolic pathways metabolism, phenylpropanoid biosynthesis, hormone biosynthesis, and expression of genes and proteins related to photosynthesis, effectively promoting the synthesis and metabolism pathways of key metabolites. This improved osmotic regulation capacity, antioxidant capacity, and water retention capacity of cells. In conclusion, we conducted an in-depth study on the physiological and biochemical processes and nanmu's transcriptional and protein expression levels in response to drought stress. The reported findings lay a foundation for the physiological and molecular mechanism of drought resistance of $P$. zhennan, thus providing a reference for further indepth analysis of the molecular mechanism of drought resistance.

\section{Methods}

\section{Plant materials and drought stress treatments}

The experiment was conducted at the Key Laboratory of Plant Resources Conservation and Germplasm Innovation in the mountainous region (Ministry of Education), Guizhou University, China. Healthy two-year-old nanmu plants with consistent growth were collected from Shiqian County (Guizhou Province). Two-year-old nanmu was obtained with permission from Shiqian County Changrong Forestry Office and transplanted into plastic pots $(24 \times 19 \times 5 \mathrm{~cm})$ filled with nutrient soil $(\mathrm{pH} 6.0,15 \%$ organic matter, and $20 \%$ humic acid). The plants were further grown under a $12 \mathrm{~h}$ light $\left(24^{\circ} \mathrm{C}\right) / 12 \mathrm{~h}$ dark $\left(20^{\circ} \mathrm{C}\right)$ photoperiod with $8000 \mathrm{Lx}-10,000 \mathrm{Lx}$ photosm ${ }^{-2} \cdot \mathrm{s}^{-2}$ light intensity and $60 \%$ relative humidity in a growth chamber (Shenzhen Samkoon Technology Corporation Ltd., China) for 16 days.

\section{Physiological and biochemical experiments}

The leaves of nanmu seedings (three biological replicates) were collected for drought stress at $0,4,8,12$, and 16 days. The RWC of leaves was calculated using the formula previously described by Ge et al. [67]. PRO, $\mathrm{Chl} \mathrm{a}$ and $\mathrm{b} \mathrm{Chl} \mathrm{b}, \mathrm{POD}, \mathrm{MDHAR}$, and GST were each assayed with PRO, Chl a/b, POD, MDHAR, H2O2, SS, O2. - and GST assay kits, respectively (Suzhou Comin Biotechnology Co. Ltd., China). ABA, SA, and JA content were measured with ultra-performance liquid chromatography-tandem MS (UPLC-MS/MS). The least significant difference (LSD) method was used to test the significance of the mean of the three replicates.

\section{Construction of transcriptome sequencing library}

The RNA-seq library preparation and sequencing were conducted by the Biomics Corporation (Beijing, China). Briefly, plants grown for 0 and $16 \mathrm{~d}$ (droughtstressed) were selected for the assay. RNA purity was verified using the NanoPhotometer ${ }^{\circledR}$ spectrophotometer (IMPLEN, CA, USA). RNA concentration was measured with the Qubit ${ }^{\circledR}$ RNA Assay Kit using the Qubit $^{\circledR}$ 2.0 Fluorometer (Life Technologies, CA, USA). After testing, the concentration and purity of the RNA were evaluated using the RNA Nano 6000 Assay Kit on the Agilent Bioanalyzer to evaluate the RNA integrity using the 2100 system (Agilent Technologies, California, USA). The library was constructed after high-quality RNA was obtained, and the qualified library was sequenced using the Illumina (New England Biolabs, USA) sequencing platform. Trinity [68] software was used to assemble the sequences after high-quality clean data were selected. The number of the read counts compared to each gene for each sample were expressed as (Fragments Per Kilobase of transcript per Million mapped reads) FPKM. The $P$-value was adjusted using the Benjamini and Hochberg method to control error discovery. Genes with adjusted $P$-values $<0.01$ identified by the DESeq2 software [69] were designated as differentially expressed. Gene functions were annotated 
based on the NCBI non-redundant protein sequences $(\mathrm{Nr})$ and Clusters of Orthologous Groups of proteins (KOG/COG). Gene Ontology (GO) enrichment analysis of the DEGs was implemented with the GOseq $R$ packages [70], which can adjust for gene length bias in DEGs. Kyoto Encyclopedia of Genes and Genomes (KEGG) [71] is a large-scale molecular database generated via genome sequencing and other high-throughput experimental technologies (http://www.genome.jp/ $\mathrm{kegg} /$ ). KOBAS [72] software was used to test the statistical enrichment of DEGs in the KEGG pathways.

\section{Protein extraction and trypsin digestion}

Plant total protein extraction was conducted according to Zhou et al. [73] method. Two hundred mg of fresh sample were weighed into a pre-cooled mortar and ground with liquid nitrogen. According to Zhao et al. [74] method, a four-fold volume of phenol extraction buffer was added for ultrasonic cracking and an equal volume of tris balanced phenol (Solarbio, China) was added for centrifugation. The supernatant was removed into a centrifuge tube for later use, and the protein concentration was determined using the BCA protein kit (Biyuntian, China). Trypsin digestion protocol was done as follows: first, the extracted proteins were reduced with $5 \mathrm{mM}$ dithiothreitol at $56^{\circ} \mathrm{C}$ for $30 \mathrm{~min}$. Next, each sample was alkylated with $11 \mathrm{mM}$ iodoacetamide and incubated for $15 \mathrm{~min}$ at room temperature in the dark. Subsequently, the sample was diluted by adding $100 \mathrm{mM}$ tetraethylammonium bromide (TEAB) to a urea concentration $<2 \mathrm{M}$. Finally, $2 \%$ trypsin was added for overnight digestion.

\section{LC-MS/MS and database analyses}

The peptides were dissolved in $0.1 \%$ formic acid (solvent A) and loaded onto the reversed-phase analytical column. The gradient was comprised of an increase from 6 to $24 \%$ of solvent B $(0.1 \%$ formic acid in acetonitrile) over $70 \mathrm{~min}, 24$ to $35 \%$ in $14 \mathrm{~min}$, increasing to $80 \%$ in $3 \mathrm{~min}$, and then holding at $80 \%$ for the last $3 \mathrm{~min}$. The flow rate was maintained at $450 \mathrm{~nL} / \mathrm{min}$. The peptides were subjected to a capillary source followed by tandem mass spectrometry (MS/MS) in tims-TOF Pro (Bruker, Germany) coupled online to the UPLC. The ion source voltage was set to $2.0 \mathrm{kV}$. The resulting MS/MS data were processed using the Maxquant search engine (v.1.6.6.0) [75]. Moreover, Correlation coefficients between the expression levels of DEPs and their corresponding mRNAs were calculated by the Pearson correlation test.

\section{Quantitative real-time PCR}

Reverse transcription of RNA was performed using the PrimeScript ${ }^{\mathrm{TM}}$ RT reagent Kit with gDNA Eraser (Perfect Real Time) (TaKaRa, Japan). The expression level of seven genes (Table S1) was verified by real-time PCR analysis using Luna ${ }^{\circledR}$ Universal qPCR Master Mix (New England Biolabs, USA) on the CFX Connect ${ }^{\mathrm{TM}}$ (BIO-RAD, USA) system. The thermocycler was set to the following program: $95^{\circ} \mathrm{C}$ for $3 \mathrm{~min} ; 95^{\circ} \mathrm{C}$ for $10 \mathrm{~s}$, 40 cycles; and $55^{\circ} \mathrm{C}$ for $20 \mathrm{~s}$. A melting curve was generated by heating the sample to $95.0^{\circ} \mathrm{C}$. The comparative $\mathrm{Ct}$ method was used to analyze the real-time PCR data [76].

\section{Abbreviations}

COG/KOG: Clusters of Orthologous Groups of proteins; KEGG: Kyoto Encyclopedia of Genes and Genomes; GO: Gene Ontology; DEGs: Differentially expressed genes; DEPs: Differentially expressed proteins; ET: Ethylene; SOD: Superoxide dismutase; CAT: Catalase; RWC: Leaf relative water content; ROS: Reactive oxygen species; PRO: Proline; MDHAR: Monodehydroascorbate reductase; GST: Glutathione S-transferase; Chl: Chlorophyll; Chl a: Chlorophyll a; Chl b: Chlorophyll b; ABA: Abscisic acid; JA: Jasmonic acid; SA: Salicylic acid; MDA: Malondialdehyde; BRs: Brassinosteroids; Od-DS: 0-day Drought-stressed; 16d-DS: 16-day Drought-stressed; O2. - : Superoxide anion; $\mathrm{H}_{2} \mathrm{O}_{2}$ : Hydrogen peroxide; SS: Soluble sugar; $\mathrm{T}^{\mathrm{U}}$ : Transcript up; $\mathrm{P}^{\mathrm{U}}$ : Protein up; $\mathrm{T}^{\mathrm{D}}$ : Transcript down; $\mathrm{P}^{\mathrm{D}}$ : Protein down.

\section{Supplementary Information}

The online version contains supplementary material available at https://doi. org/10.1186/s12870-022-03474-3.

Additional file 1: Figure S1. Sequence length distribution diagram and classification diagram. (a) Sequence length distribution diagram of assembly results. (b) NR annotated species classification map. (c) KOG annotation classification Figure S2. Real-time quantitative PCR analysis of drought stress. Figure S3. Basic statistical graph of mass spectra data results. Figure S4. Bubble charts provide the results for the top 20 categories of most significant enrichments. (a) Biological Process. (b) Cellular Component. (c) Molecular Function. (d) Protein domain enrichment. Figure S5. A scatter plot of the transcript and its corresponding protein expression. Figure S6. KEGG pathway results. Figure S7. Heatmap of DEGs and DEPs related to HSPs in Nanmu. Table S1. A detailed list of primer sequences used in this study. Table S2. The statistical results compared with the reference sequence. Table S3. Statistical table of assembly results. Table S4. Overlap of DEGs and DEPs in nanmu (genotype).

\section{Acknowledgments}

Not applicable.

\section{Authors' contributions}

DZ conceived the idea and designed the experiments. NX dealt with drought stress materials. BL did the QRT-PCR experiment. QZ and RXS measured the enzyme activity experiment. NX analyzed the RNA-seq and iTRAQ data.DZ wrote the first draft. YL and JY finalized the manuscript. All authors read and approved the final manuscript.

\section{Funding}

This research was funded by grants from Efficient Breeding Technology and Demonstration of Phoebe zhennan in Guizhou, China (NO.[2020]1Y063),

Talent Base for Germplasm Resources Utilization and Innovation of Characteristic Plant in Guizhou (No.RCJD2018-14), Research on Excellent Seed and Seedling Breeding of Guizhou Rare Tree P. zhennan (No. (2018) 05), the study on the molecular regulatory networks of $P$. zhennan polyembryony based on 
multi-omics analysis (No. YJSCXJH[2020]081), and the study on the techniques of sexual propagation and transformation of low-quality and low-efficiency forest of P. zhennan (young forest) (Guizhou) (No. 2017YFD0601102).

\section{Availability of data and materials}

The data sets generated and analyzed in our study are available in the NCBI Sequence Read Archive under the accession number of RJNA778346.

\section{Declarations}

\section{Ethics approval and consent to participate}

The experimental research on plants (including seeds) performed in this study complies with institutional, national and international guidelines.

\section{Consent for publication}

Not applicable.

\section{Competing interests}

The authors declare that they have no competing interests.

\section{Author details}

${ }^{1}$ Institute of Agro-Bioengineering and College of Life Sciences, The Key Laboratory of Plant Resources Conservation and Germplasm Innovation in Mountainous Region (Ministry of Education), Guizhou University, Guiyang 550025, Guizhou, China. ${ }^{2}$ Guizhou Academy of Agricultural Sciences, Guizhou Plant Conservation Technology Center, Guiyang 550006, Guizhou, China. ${ }^{3}$ Tobacco Molecular Genetics Key Laboratory of China Tobacco, Guizhou Academy of Tobacco Science, Guiyang 550081, China. ${ }^{4}$ Guizhou Academy of Forestry, Guiyang 550005, Guizhou, China.

\section{Received: 2 November 2021 Accepted: 15 February 2022} Published online: 03 March 2022

\section{References}

1. $\mathrm{Hu} \mathrm{H}$, Xiong L. Genetic engineering and breeding of drought-resistant crops. Annu Rev Plant Biol. 2014;65:715-41.

2. Uga Y, Sugimoto K, Ogawa S, Rane J, Ishitani M, Hara N, et al. Control of root system architecture by DEEPER ROOTING 1 increases rice yield under drought conditions. Nat Genet. 2013;45(9):1097-102.

3. Comas LH, Becker SR, Cruz V, Byrne PF, Dierig DA. Root traits contributing to plant productivity under drought. Front Plant Sci. 2013;4:442.

4. Xiong L, Schumaker KS, Zhu JK. Cell signaling during cold, drought, and salt stress. Plant Cell. 2002;14(Suppl):S165.

5. Verslues PE, Agarwal M, Katiyar-Agarwal S, Zhu J, Zhu JK. Methods and concepts in quantifying resistance to drought, salt and freezing, abiotic stresses that affect plant water status. Plant J. 2006;45(4):523-39.

6. Gao JH, Zhang W, Li JY, Long HL, He W. Amplified fragment length polymorphism analysis of the population structure and genetic diversity of Phoebe zhennan (Lauraceae), a native species to China. Biochem Syst Ecol. 2016;64:149-55.

7. Chaves MM, Maroco JP, Pereira JS. Understanding plant responses to drought — from genes to the whole plant. Funct Plant Biol. 2003;30(3):239-64.

8. El-Sharkawy MA. Cassava biology and physiology. Plant Mol Biol. 2004;56(4):481-501.

9. Okogbenin E, Setter TL, Ferguson M, Mutegi R, Ceballos H, Olasanmi B, et al. Phenotypic approaches to drought in cassava: review. Front Plant Sci. 2013;4:93.

10. Sharma M, Gupta SK, Majumder B, Maurya VK, Pandey V. Salicylic acid mediated growth, physiological and proteomic responses in two wheat varieties under drought stress. J Proteome. 2017;163:28.

11. Impa SM, Nadaradjan S, Jagadish S. Drought stress induced reactive oxygen species and anti-oxidants in plants. New York: Springer; 2012. p. $131-47$.

12. Li ZG, Yuan LX, Wang QL, Ding ZL. Combined action of antioxidant defense system and osmolytes in chilling shock-induced chilling tolerance in Jatropha curcas seedlings. Acta Physiol Plant. 2013;35(7):2127-36.
13. Mohammadkhani N, Heidari R. Drought-induced accumulation of soluble sugars and Proline in two maize varieties. World Appl Sci. 2008;3(3):448-53.

14. Li D, Li C, Sun H, Wang W, Liu L, Zhang Y. Effects of drought on soluble protein content and protective enzyme system in cotton leaves. Front Agric China. 2010;4:56-62.

15. Ge T, Sui F, Bai L, Lu Y, Zhou G. Effects of water stress on the protective enzyme activities and lipid peroxidation in roots and leaves of summer maize. Agric Sci China. 2006;5:291-8.

16. Zeng D. Physiological effects of chitosan coating on wheat growth and activities of protective enzyme with drought tolerance. Open J Soil Sci. 2012;02(3):282-8

17. Savoi S, Wong D, Arapitsas P, Miculan M, Bucchetti B, Peterlunger E, et al. Transcriptome and metabolite profiling reveals that prolonged drought modulates the phenylpropanoid and terpenoid pathway in white grapes (Vitis vinifera L.). BMC Plant Biol. 2016;16(1):67.

18. Shinozaki K, Yamaguchi-Shinozaki K. Gene networks involved in drought stress response and tolerance. J Exp Bot. 2007;58:221-7.

19. Roychoudhury A, Paul S, Basu S. Cross-talk between abscisic aciddependent and abscisic acid-independent pathways during abiotic stress. Plant Cell Rep. 2013;32(7):985-1006.

20. Kumar J, Abbo S. Genetics of flowering time in chickpea and its bearing on productivity in semiarid environments. Adv Agron. 2001;72(2):107-38.

21. Fu XZ, Tong YH, Zhou X, Ling LL, Chun CP, Cao L, et al. Genome-wide identification of sweet orange (Citrus sinensis) metal tolerance proteins and analysis of their expression patterns under zinc, manganese, copper, and cadmium toxicity. Gene. 2017;629:1-8.

22. Cattivelli L, Rizza F, Badeck FW, Mazzucotelli E, Mastrangelo AM, Francia E, et al. Drought tolerance improvement in crop plants. An integrated view from breeding to genomics. Field Crop Res. 2008;105(1-2):1-14.

23. Zhu JK. Abiotic stress signaling and responses in plants. Cell. 2016;167:313-24

24. Ma X, Wang P, Zhou SH, Zhou SH, Sun Y, Liu NN. De novo transcriptome sequencing and comprehensive analysis of the drought-responsive genes in the desert plant Cynanchum komarovii. BMC Genomics. 2015;16:753.

25. Ding Z, Fu L, Tie W, Yan Y, Wu C, Hu W, et al. Extensive post-transcriptional regulation revealed by Transcriptomic and proteomic integrative analysis in cassava under drought. J Agric Food Chem. 2019;67:3521-34.

26. Zhao Y, Gao C, Shi F, Yun L, Jia Y, Wen J. Transcriptomic and proteomic analyses of drought responsive genes and proteins in Agropyron mongolicum Keng. Curr Plant Biol. 2018;14:19-29.

27. Tariq A, Pan K, Olatunji OA, Graciano C, Li N, Li Z, et al. Role of nitrogen supplementation in alleviating drought-associated growth and metabolic impairments in Phoebe zhennan seedlings. J Plant Nutr Soil Sci. 2019;182:586-96.

28. Hu Y, Wang B, Hu T, Chen H, Li H, Zhang W, et al. Combined action of an antioxidant defence system and osmolytes on drought tolerance and post-drought recovery of Phoebe zhennan S. Lee saplings. Acta Physiol Plant. 2015;37(4):84

29. Bartlett MK, Scoffoni C, Sack L. The determinants of leaf turgor loss point and prediction of drought tolerance of species and biomes. A global meta-analysis. Ecol Lett. 2015;15(5):393-405.

30. Gong Z, Xiong L, Shi H, Yang S, Zhu JK. Plant abiotic stress response and nutrient use efficiency. Sci China Life Sci. 2020;63:5.

31. Zhao TJ, Sun S, Liu Y, Liu JM, Zhou HM. Regulating the DRE-mediated signaling pathway by synergic functions of trans-active and trans-inactive DREBs in brassica Napus. J Biol Chem. 2006;281(16):10752-9.

32. Han J. The Responses of Rice (Oryza sativa L.) Seedlings to the Osmotic Stress and Relation to the Osmotic Adjustment. J Agric Univ Hebei. 1990;1:117-21.

33. Regan KL, Whan BR, Turner NC. Evaluation of chemical desiccation as a selection technique for drought resistance in a dryland wheat breeding program. Crop Pasture Sci. 1993;44(8):1683-91.

34. Faize M, Burgos L, Faize L, Piqueras A, Nicolas E, Ba R, et al. Involvement of cytosolic ascorbate peroxidase and cu/Zn-superoxide dismutase for improved tolerance against drought stress. J Exp Bot. 2011;62(8):2599.

35. Wei L, He Y, Xiang J, Fu C, Li M. The physiological response of suspension cell of Capparis spinosa $L$ to drought stress. J Med Plant Res. 2011;5(24):5899-906. 
36. Silva EN, Ferreira-Silva SL, Viégas R, Silveira J. The role of organic and inorganic solutes in the osmotic adjustment of drought-stressed Jatropha curcas plants. Environ Exp Bot. 2010;69(3):279-85.

37. Reddy AR, Chaitanya KV, Vivekanandan M. Drought-induced responses of photosynthesis and antioxidant metabolism in higher plants. J Plant Physiol. 2004;161(11):1189-202.

38. Sorkheh S, Rouhi K, Sofo. Regulation of the ascorbate-glutathione cycle in wild almond during drought stress. Russ J Plant Physl+. 2011;58(1):76-84.

39. Rezaei MK, Shobbar Z-S, Shahbazi M, Abedini R, Zare S. Glutathione S-transferase (GST) family in barley: identification of members, enzyme activity, and gene expression pattern. J Plant Physiol. 2013;170(14):1277-84.

40. Wu H, Wu X, Li Z, Duan L, Zhang M. Physiological evaluation of drought stress tolerance and recovery in cauliflower ( Brassica oleracea L.) seedlings treated with methyl Jasmonate and Coronatine. J Plant Growth Regul. 2012;31(1):113-23.

41. Anjum F, Yaseen M, Rasool E, Wahid A, Anjum S. Water stress in barley (Hordeum vulgare L.). II. Effect on chemical composition and chlorophyll contents. Pak J Agric Sci. 2003;26(9):203-9.

42. Fraser CM, Chapple C. The Phenylpropanoid pathway in Arabidopsis. Arabidopsis Book. 2011;9:e0152.

43. Harb K. Ambavaram, MMR, Pereira: molecular and physiological analysis of drought stress in Arabidopsis reveals early responses leading to acclimation in plant growth. Plant Physiol. 2010;154:1254-71.

44. Morgan PW. Effects of abiotic stresses on plant hormone systems; 1990 . p. 12.

45. Daszkowska-Golec A. The role of abscisic acid in drought stress: how ABA helps plants to cope with drought stress. In: Hossain MA, Wani SH, Bhattacharjee $\mathrm{S}$, editors. Drought stress tolerance in plants, Molecular and Genetic Perspectives. Cham: Springer International Publishing; 2016. p. 123-51.

46. Negin B, Moshelion M. The evolution of the role of ABA in the regulation of water-use efficiency. From biochemical mechanisms to stomatal conductance. Plant Sci. 2016;251:82-9.

47. Akash T, Pan K, Olatunji OA, Corina G, Li Z, Feng S, et al. Phosphorous application improves drought tolerance of Phoebe zhennan. Front Plant Sci. 2017:8:1561

48. McAdam SAM, Brodribb TJ. Linking turgor with ABA biosynthesis: implications for Stomatal responses to vapor pressure deficit across land plants. Plant Physiol. 2016;171(3):2008-16.

49. Audran C, Liotenberg S, Gonneau M, North H, Marion-Poll A. Localisation and expression of zeaxanthin epoxidase mRNA in Arabidopsis in response to drought stress and during seed development. Austjplant Physiol. 2001:28(12):1161-73.

50. Anstis P, Friend J, Gardner D. The role of xanthoxin in the inhibition of pea seedling growth by red light. Phytochemistry. 1975;14(1):31-5.

51. Koornneef M, Léon-Kloosterziel K, Schwartz SH, Zeevaart J. The genetic and molecular dissection of abscisic acid biosynthesis and signal transduction in Arabidopsis. Plant Physiol Biochem. 1998;36(1-2):83-9.

52. Bittner $F$, Oreb M, Mendel RR. ABA3 is a molybdenum cofactor Sulfurase required for activation of aldehyde oxidase and xanthine dehydrogenase in Arabidopsis thaliana. J Biol Chem. 2001;276(44):40381-4.

53. Bing-Qin XU, Gao XL, Gao JF, Jing LI, Yang P, Feng BL. Transcriptome profiling using RNA-seq to provide insights into foxtail millet seedling tolerance to short-term water deficit stress induced by PEG-6000. J Integr Agric. 2019;18(11):2457-71.

54. Zhou XE, Soon FF, Ng LM, Kovach A, Suino-Powell KM, Li J, et al. Catalytic mechanism and kinase interactions of ABA-signaling PP2C phosphatases. Plant Signal Behav. 2014;7(5).

55. Szostkiewicz I, Richter K, Kepka M, Demmel S, Grill E. Closely related receptor complexes differ in their ABA selectivity and sensitivity. Plant J. 2009:61(1):25-35.

56. Chen K, Li G, Bressan RA, Song C, Zhu J, Zhao Y. Abscisic acid dynamics, signaling, and functions in plants. J Integr Plant Biol. 2020;62(01):25-54.

57. Grondin A, Mauleon R, Vadez V, Henry A. Root aquaporins contribute to whole plant water fluxes under drought stress in rice (Oryza sativa L.). Plant Cell Environ. 2016;39:347-65.

58. Jin M. The role of PIP aquaporins in response to various environmental scenarios in Arabidopsis thaliana; 2015.
59. Alexandersson E, Fraysse L, Sjvall-Larsen S, Gustavsson S, Fellert M, Karlsson $\mathrm{M}$, et al. Whole gene family expression and drought stress regulation of Aquaporins. Plant Mol Biol. 2005;59(3):469-84.

60. Lee J, Parthier B, Lobler M. Jasmonate signalling can be uncoupled from abscisic acid signalling in barley. Identification of jasmonateregulated transcripts which are not induced by abscisic acid. Planta. 1996;199(4):625-32

61. Dong T, Cai K, Zhang J, Rong H, Xie G. The physiological roles of methyl jasmonate (MeJA) in drought resistance of rice seedlings. Ecol Environ. 2007;16:1261-5.

62. Rohit D, Raorane ML, Achim T, Peethambaran PK, Schendel RR, Sahi VP, et al. Corrigendum: Physiological and proteomic analysis of the Rice mutant cpm2 suggests a negative regulatory role of Jasmonic acid in drought tolerance. Front Plant Sci. 2018;9:465.

63. Timperio AM, Egidi MG, Zolla L. Proteomics applied on plant abiotic stresses: role of heat shock proteins (HSP). J Proteome. 2009;71(4):391-411.

64. Clément M, Leonhardt N, Droillard MJ, Reiter I, Montillet $\mathrm{L}$, Genty B, et al. The cytosolic/nuclear hsc70 and hsp90 molecular chaperones are important for stomatal closure and modulate abscisic acid-dependent physiological responses in Arabidopsis. Plant Physiol. 2011;156(3):1481-92.

65. Reinbothe C, Bakkouri ME, Buhr F, Muraki N, Nomata J, Kurisu G, et al. Chlorophyll biosynthesis: spotlight on protochlorophyllide reduction. Trends Plant Sci. 2010;15(11):614-24.

66. Xiao B, Huang Y, Ning T, Xiong L. Over-expression of a LEA gene in rice improves drought resistance under the field conditions. Theor Appl Genet. 2007;115(1):35-46.

67. Ge Y, He X, Wang J, Jiang B, Ye R, Lin X. Physiological and biochemical responses of Phoebe bournei seedlings to water stress and recovery. Acta Physiol Plant. 2014;36(5):1241-50.

68. Grabherr MG, Haas BJ, Yassour M, Levin JZ, Amit I. Trinity:reconstructing a full-length transcriptome without a genome from RNA-Seq data. Nat Biotechnol. 2013;29:644.

69. Wang L, Feng Z, Wang X, Wang X, Zhang X. DEGseq: an R package for identifying differentially expressed genes from RNA-seq data. Bioinformatics. 2010;26:136-8.

70. Young M, Wakefield MJ, Smyth GK, Oshlack A. Gene ontology analysis for RNA-seq: accounting for selection bias. Genome Biol. 2010;11(2):R14.

71. Minoru K, Michihiro A, Susumu G, Masahiro H, Mika H, Masumi I, et al. KEGG for linking genomes to life and the environment. Nucleic Acids Res. 2008:36:480-4

72. Mao X, Tao C, Olyarchuk JG, Wei L. Automated genome annotation and pathway identification using the KEGG Orthology (KO) as a controlled vocabulary. Bioinformatics. 2005;21(19):3787-93.

73. Zhou H, Finkemeier I, Guan W, Tossounian MA, Wei B, Young D, et al. Oxidative stress-triggered interactions between the succinyland acetylroteomes of rice leaves. Plant Cell Environ. 2018:41:1139-53.

74. Zhao D, Xie N, Zhao D. Analysis of acetylated proteomics in response to drought stress of Phoebe zhennan leaves. Plant Physiol J. 2021;57:1371-81

75. Liu Y, Zhang Z, Fan H, Tan Y, Xu H. Phosphoproteomics of cold stressresponsive mechanisms in Rhododendron Chrysanthum. Res Square. 2021;49(1).

76. Schefe JH, Lehmann KE, Buschmann IR, Unger T, Funkekaiser H. Quantitative real-time RT-PCR data analysis: current concepts and the novel'gene expression's CT difference' formula. J Mol Med. 2006;84:901-10.

\section{Publisher's Note}

Springer Nature remains neutral with regard to jurisdictional claims in published maps and institutional affiliations. 